\title{
Estimación del riesgo de crédito en proyectos de infraestructura mediante modelos estructurales
}

\author{
Estimation of credit risk in infrastructure projects using structural models
}

\section{Carlos Andrés Zapata Quimbayo*}

Universidad Externado de Colombia, Colombia

Recibido el 28 de marzo de 2019; aceptado el 2 de diciembre de 2019

Disponible en Internet el: 14 de enero de 2020

\section{Resumen}

Este trabajo tiene como objetivo implementar un modelo de riesgo de crédito en proyectos de infraestructura, donde se estima la probabilidad de incumplimiento teniendo en cuenta el flujo de efectivo disponible para el servicio de deuda y su ratio de cobertura. Para ello, se utiliza un modelo estructural de riesgo de crédito desarrollado para activos ilíquidos, como una extensión de los modelos de Merton (1974) y KMV de Moody's, mediante el cual se analizan los componentes de probabilidad de incumplimiento, exposición, tasa de recuperación y pérdida esperada. La principal innovación de este enfoque se debe a la incorporación de una dinámica propia de la ratio de cobertura del servicio de deuda, el cual se modela estocásticamente siguiendo los mismos supuestos de la teoría de valoración de opciones financieras. Además, este modelo se complementa con la técnica de simulación de Monte Carlo, bajo la cual se estiman algunos parámetros esenciales, así como la pérdida esperada y el valor en riesgo $(\mathrm{VaR})$ crediticio.

Código JEL: C14, G13, G21

Palabras clave: Riesgo de crédito; Probabilidad de incumplimiento; Proceso estocástico

\footnotetext{
*Autor para correspondencia Correo electrónico carlosa.zapata@uexternado.edu.co (C.A. Zapata Quimbayo). La revisión por pares es responsabilidad de la Universidad Nacional Autónoma de México. 


\begin{abstract}
This paper aims to implement a credit risk model in infrastructure investment projects, where the probability of default is estimated considering the cash flow available for debt service, which determines the debt service coverage ratio. For that, a structural model developed for illiquid assets is used, such as an extension of the credit risk models of Merton (1974) and KMV of Moody's, through which the components of the probability of default, exposure, recovery rate and expected loss are analyzed. The main innovation of this approach is due to the incorporation of a dynamic of the debt service coverage ratio, which is modelled stochastically following the same assumptions of the option pricing theory. In addition, this model is complemented with the Monte Carlo simulation technique, under which some essential parameters are estimated, as well as the expected loss and the credit value-at-risk (VaR).
\end{abstract}

JEL code: $\mathrm{C} 14, \mathrm{G} 13, \mathrm{G} 21$

Keywords: Credit risk; Probability of default; Stochastic process

\title{
Introducción
}

La financiación de proyectos, conocida en la teoría financiera como Project Finance, comprende el diseño de una compleja estructura de financiación que vincula a inversionistas privados con el propósito de financiar, construir y gestionar complejas infraestructuras, generalmente de carácter público (Grimsey y Lewis, 2002). El principal objetivo de este esquema ha sido el de aumentar la eficiencia del Gobierno, mediante la generación de incentivos ${ }^{1}$, así como la asignación y transferencia de riesgos ${ }^{2}$ (Yescombe, 2002; Gatti, 2008). Bajo este esquema, se crea un vehículo de propósito especial (SPV, por sus siglas en inglés) sobre una base ad hoc de carácter independiente respecto a los patrocinadores y con recursos limitados de estos.

Además, según Gatti (2008), la financiación de proyectos cuenta con características distintivas en comparación al financiamiento corporativo tradicional, como: i) es una forma estructurada de financiación fuera de balance; ii) el SPV requiere de un nivel de especificidad que determina su propósito y naturaleza asociativa; iii) los recursos de los patrocinadores son limitados o nulos, lo cual representa un alto nivel de apalancamiento con largos plazos

\footnotetext{
${ }^{1}$ Las limitaciones de recursos públicos para la inversión en infraestructura, las restricciones en su capacidad de endeudamiento, principalmente en los países en vía de desarrollo, junto con la necesidad de mejorar y ampliar la provisión de bienes y servicios públicos, ha llevado a que los gobiernos acudan ante inversionistas privados para participar en acuerdos contractuales de largo plazo denominados acuerdos de asociación público-privada (APP) e iniciativas de financiación privada (IFP).

${ }^{2}$ La asignación de los riesgos del proyecto entre los diversos participantes de la financiación es crucial para determinar su éxito. De igual forma, la financiación de proyectos se fundamenta en el establecimiento de una compleja red de contratos en la que se identifican diferentes partes involucradas con distintos roles y, donde los contratos celebrados por el SPV no solo funcionan como garantía para acceder al financiamiento, sino que permiten la transferencia de los riesgos a la contraparte que mejor los puede gestionar.
} 
para recuperar la inversión; y iv) la financiación incorpora derechos de control significativos y extensos para prestamistas, así como covenants y clausulas restrictivas.

De esta forma, todas las consecuencias económicas del proyecto son atribuidas directamente al SPV. Por tal motivo, el reembolso de la deuda depende principalmente de la capacidad del proyecto para generar flujo de efectivo ${ }^{3}$, lo cual determina su capacidad crediticia. Los flujos de efectivo se consideran como la principal fuente de recursos para cubrir las obligaciones de deuda contraídas, es decir, una vez el proyecto ha cubierto sus requerimientos operativos (costos, mantenimiento, gastos, impuestos, entre otros) a partir de los ingresos generados. Así, el efectivo disponible debe ser suficiente para pagar el servicio de deuda (capital más intereses). A esto se suma la ausencia de antecedentes crediticios del proyecto que sirvan como base para una decisión de financiación como indica Cappon et al. (2018).

Bajo estas características, el análisis crediticio debe determinar si el vehículo de inversión este estará o no en capacidad de pagar la deuda contraída. El problema radica en la incertidumbre futura sobre el éxito del proyecto, dado los diferentes riesgos que están presentes en sus fases del ciclo de vida, por ejemplo, una demora en la terminación de la construcción por eventos naturales o sociales, una reducción de su demanda o de sus ingresos, un aumento de los costos operativos, entre otros (Cartea y Figueroa, 2005; Boussabaine, 2014). Además, cualquier incumplimiento de las obligaciones contractuales de las contratistas puede interrumpir el correcto funcionamiento del proyecto. Esta incertidumbre se traduce en la probabilidad de incumplir el acuerdo de financiación ${ }^{4}$, es decir un incumplimiento de los pagos del servicio de deuda, ya sea parcial o totalmente.

Por esta razón, el otorgamiento de deuda en proyectos de infraestructura, principalmente de iniciativa privada, han incorporado derechos de control (significativos y extensos) para los prestamistas (Borgonovo y Gatti, 2013; Blanc-Brude, Hasan e Ismail, 2014), así como covenants y clausulas restrictivas (Gatti, 2008; Blanc-Brude, Hasan y Whittaker, 2016), al tiempo que se prohíben nuevas emisiones de deuda para pagar la deuda existente. Por lo tanto, el análisis crediticio en proyectos de infraestructura termina siendo mucho más complejo (Jobst, 2018; Wang et al., 2019), en comparación con el sector corporativo.

Como resultado, un análisis rigoroso del riesgo de crédito requiere reflejar estas características. Sin embargo, los modelos tradicionales de evaluación crediticia: i) modelos de forma reducida; ii) modelos basados en calificaciones y, iii) modelos estructurales, no pueden

\footnotetext{
${ }_{3}^{3}$ De acuerdo con Boussabaine (2014), una característica estructural del SPV es su limitada discreción en la gestión del proyecto al enfocar todos sus esfuerzos en la generación del flujo de efectivo, con los cuales busca cubrir las obligaciones contraídas y remunerar a todas las partes involucradas.

${ }^{4} \mathrm{Al}$ respecto, Jobst (2018) encuentra que los proyectos de infraestructura alcanzan una tasa de recuperación cercana al $80 \%$ (en promedio).
} 
aplicarse directamente o presentan fuertes limitaciones para este tipo de financiaciones, como se ha encontrado en trabajos previos desarrollados por Klompjan y Wouters (2002), Gatti et al. (2007), Kong et al. (2008), Dong et al. (2012), Karminsky y Morgunov (2016), Wang et al. (2019), entre otros. Además, Klompjan y Wouters (2002) y Karminsky y Morgunov (2016) señalan que hay limitaciones asociadas a la poca disponibilidad de datos sobre incumplimientos (históricos) y/o a las dificultades de acceso a esta información, así como a la diversidad en el propósito de cada proyecto y de sus riesgos, lo cual dificulta la determinación de las variables explicativas.

A parte de estas limitaciones, se encuentra que los modelos anteriores no permiten incorporar, no solo los efectos de los covenants de deuda, sino también la naturaleza dinámica de la capacidad de pago del proyecto como indican Blanc-Brude et al. (2016). Aunque este último inconveniente es superado el campo de los modelos estructurales, como muestran Freydefornt (2001) y Aragones, Blanco e Iniesta (2009), quienes implementaron modelos de estimación del riesgo de crédito en proyectos de infraestructura basados en los modelos de Merton (1974) y KMV de Moody's, aún persisten limitaciones. Como respuesta, Blanc-Brude y Hasan (2016) proponen una extensión de modelo KMV de Moody's para estimar la probabilidad de incumplimiento en proyectos de infraestructura. A diferencia de las aplicaciones anteriores, ellos estiman la probabilidad de incumplimiento a partir de un tratamiento estocástico en la dinámica del perfil pago del proyecto, el cual se determina a través del efectivo disponible. Este modelo fue extendido por Blanc-Brude et al. (2016) para estimar la probabilidad condicional de transición entre estados (de un estado riesgoso a uno seguro y, viceversa) al integrar técnicas de inferencia bayesiana.

De igual forma, Wang et al. (2019) proponen un modelo CreditMetrics ajustado acompañado con simulación de Monte Carlo para estimar el riesgo de crédito basado en la información que proporcionan los flujos de efectivo del proyecto. A partir de un análisis cualitativo apoyado en la "escala de recuperación" de Standard \& Poor's superan las limitaciones asociadas a la falta de información y logran estimar la pérdida esperada y sus componentes. Sin embargo, esta aplicación presenta sesgos al incorporar elementos subjetivos en la estimación, los cuales son resaltados por los mismos autores.

Partiendo de estos desarrollos, este trabajo tiene como objetivo implementar un modelo de estimación del riesgo de crédito, donde se estima la probabilidad de incumplimiento en proyectos de infraestructura al incorporar la dinámica del perfil de pago usando el flujo de efectivo disponible y la ratio de cobertura del servicio de deuda. Para ello, se adopta un modelo estructural de riesgo de crédito desarrollado para activos ilíquidos siguiendo los trabajos de 
Blanc-Brude y Hasan (2016) y Blanc-Brude et al. (2016). De igual forma, se analiza la exposición y pérdida potencial para un proyecto hipotético de concesión de una carretera de peaje. Esta metodología representa una extensión de los modelos tradicionales de riesgo de crédito de Merton (1974) y KMV de Moody's y, la principal innovación de este enfoque se debe a la incorporación de una dinámica propia de la ratio de cobertura del servicio de deuda (DSCR, por su sigla en inglés), el cual se modela estocásticamente siguiendo los mismos supuestos de la teoría de valoración de opciones financieras. Además, este modelo complementa con la técnica de simulación de Monte Carlo, bajo la cual se estiman algunos parámetros esenciales, así como la pérdida esperada y el valor en riesgo ( $\mathrm{VaR})$.

\section{Modelos estructurales de riesgo de crédito}

A partir de los trabajos seminales de Black y Scholes (1973) y Merton (1973), los modelos de valoración de opciones financieras han sido adaptados para el tratamiento de problemas corporativos, entre ellos la evaluación del riesgo de crédito. Estos desarrollos se encuentran inicialmente en los trabajos de Merton (1974) y Merton (1977), así como en las extensiones realizadas por Black y Cox (1976) e Ingersoll (1977). Todos estos desarrollos se identifican, para efectos de este trabajo, como el "modelo de Merton". Este campo comprende el inicio del enfoque de estimación de riesgo de crédito a partir de modelos estructurales ${ }^{5}$, lo cuales se presentan a continuación.

\section{Modelo de Merton}

Merton (1974) extendió la fórmula de Black-Scholes al análisis de reclamaciones contingentes (CCA, por su sigla en inglés) para el tratamiento de problemas corporativos, donde asume que la deuda de una empresa puede ser considerada como una reclamación sobre los activos de esta, con un precio de ejercicio igual a su valor nominal y una fecha de vencimiento determinada. De esta forma, propone una relación entre la estructura de capital y la capacidad que tiene la empresa para pagar su deuda. Para ello, asume que la evolución del valor de mercado de los activos de la empresa $\left(V_{A}\right)$ sigue un proceso estocástico del tipo movimiento Browniano geométrico $(\mathrm{mBg})$ que viene dado por la ecuación 1.

$$
d V_{A}=\mu_{A} V_{A} d t+\sigma_{A} V_{A} d W_{t}
$$

\footnotetext{
${ }^{5}$ A diferencia de los modelos de forma reducida y aquellos basados en calificaciones, en los modelos estructurales se asume que los inversores tienen información completa del mercado, por tanto, estos tienen conocimiento del valor de los activos y la deuda de las empresas.
} 
Donde, $\mu_{A}$ representa a la tasa de deriva del valor de los activos y $\sigma_{A}$ su volatilidad, $W_{t \in[0, T]}$ es un proceso estándar de Wiener definido en un espacio de probabilidad $(\Omega, \mathcal{F}, P))$ con una filtración $\left(\mathcal{F}_{t}\right)_{t \in[0, T] \text {. }}$

El valor de los activos de la empresa está determinado por $V_{A}=V_{E}+V_{D}$ en un momento inicial $\mathrm{t}=0$, donde $V_{E}$ es el valor de mercado de las acciones de la empresa, y $V_{D}$ es el valor de su deuda. Además, se asume que $V_{D}$ está representada por la emisión de un bono cero cupón con vencimiento en $T$ y con valor $B(t, T)$. Entonces, si $V_{A}<V_{D}$ entonces la empresa incumple el pago de su deuda, en este caso $V_{E}=0$, mientras que si $V_{A}>V_{D}$, la empresa paga su deuda en $T$ y, por tanto, $V_{E}=V_{A}-V_{D}$. Esta lógica se puede representar como una función de la forma:

$$
V_{E}=\max \left(V_{A}-V_{D}, 0\right)
$$

De esta forma, $V_{E}$ se asimila a una opción de compra de tipo europea con precio de ejercicio igual $V_{D}$. Lo anterior indica que, si el valor de los activos es insuficiente para cumplir con la deuda, entonces los accionistas, quienes son titulares de la opción de compra, no ejercerán su derecho y dejarán la empresa a sus acreedores.

Ahora, la ecuación 1 se puede representar en términos logarítmicos, por tanto, se tiene:

$$
\ln V_{A}=\ln V_{0}+\left(\mu-\frac{1}{2} \sigma_{A}^{2}\right) t+\sigma_{A} Z \sqrt{T}
$$

Donde $Z \sim N(0,1)$ y, la probabilidad de incumplimiento de la empresa $(P(t, T))$ entre $t$ y $T$ $\mathrm{y}$, viene dada por

$$
P(t, T)=P\left[V_{A} \leq V_{D}\right]=P\left[\ln V_{A} \leq \ln V_{D}\right]
$$

Entonces, en un mundo riesgo-neutral, el valor de $V_{E}$ está determinado por la fórmula

$$
V_{E}=V_{A} N\left(d_{1}\right)-V_{D} e^{-r T} N\left(d_{2}\right)
$$

Donde $r$, representa la tasa de interés libre de riesgo y $N(\cdot)$ indica la función de distribución normal acumulada de los parámetros $d_{1}$ y $d_{2}$, como muestran las ecuaciones $6 \mathrm{a}$ y $6 \mathrm{~b}$.

$$
d_{1}=\frac{\ln \left(\frac{V_{A}}{V_{D}}\right)+\left(r+\frac{1}{2} \sigma_{A}^{2}\right) T}{\sigma_{A} \sqrt{T}}
$$




$$
d_{2}=\frac{\ln \left(\frac{V_{A}}{V_{D}}\right)+\left(r-\frac{1}{2} \sigma_{A}^{2}\right) T}{\sigma_{A} \sqrt{T}}=d_{1}-\sigma_{A} \sqrt{T}
$$

Finalmente, la probabilidad de incumplimiento (riesgo-neutral) es la probabilidad de que $V_{A}$ en $T$ este por debajo de $V_{D}$. Entonces se tiene

$$
P\left[V_{A} \leq V_{D}\right]=N\left(-d_{2}\right)
$$

Una limitación importante del modelo de Merton es que los parámetros del proceso determinado por la ecuación 1 , es decir, $\mu_{A} \mathrm{y} \sigma_{A}$, no son directamente observables, lo cual dificulta su aplicación.

\section{Modelo KMV de Moody's}

El modelo KMV de Moody's fue desarrollado inicialmente por Vasicek (1984) y, posteriormente complementado por McQuown (1993) y Kealhofer (1993), como una extensión del modelo Merton para estimar la probabilidad de incumplimiento de una empresa basado en la noción de distancia al incumplimiento (DD, por su sigla en inglés). A diferencia del modelo de Merton, el modelo KMV asume que la empresa cae en incumplimiento cuando el valor de sus activos está por debajo de un umbral definido por el valor de su deuda, la cual determina el punto de incumplimiento.

La figura 1, muestra su aplicación a partir de la representación de una trayectoria posible del valor de mercado de los activos. Allí, se observa que, si el valor de los activos cae por debajo del punto de incumplimiento, entonces la empresa no tiene como pagar la deuda. Por lo tanto, la probabilidad de incumplimiento es la probabilidad de que el valor del activo se encuentre por debajo de este punto. Esta probabilidad está representada en el área sombreada de la función de distribución debajo del punto de incumplimiento. 


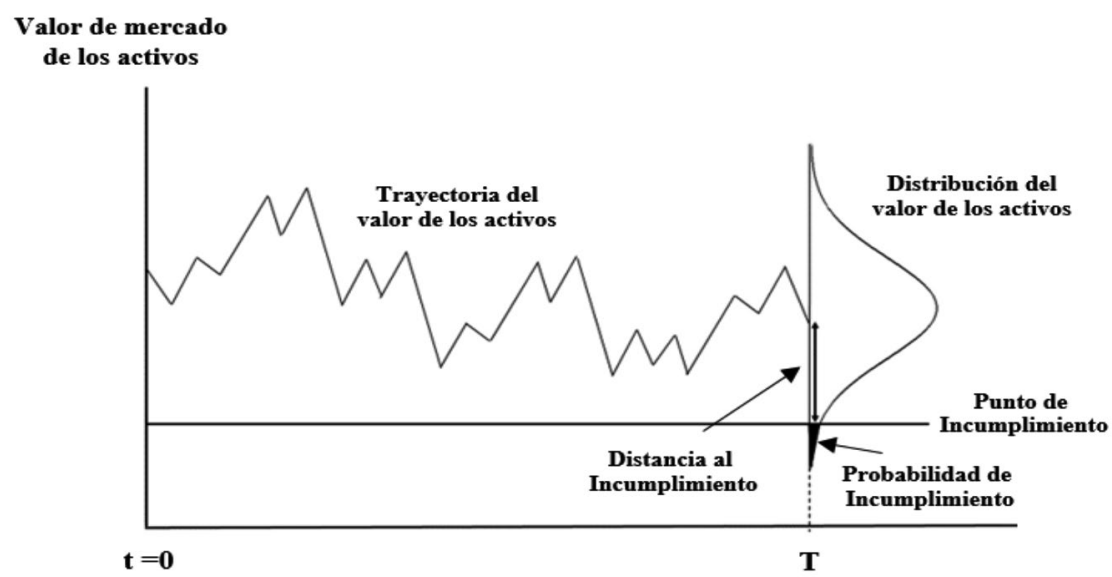

Figura 1. Representación del incumplimiento a partir de la distancia al incumplimiento Fuente: adaptado de Kealhofer (2003).

Como resultado, el modelo estima la distancia al incumplimiento como el número de desviaciones estándar en que el valor de los activos supera el punto de incumplimiento. Entonces, la distancia al incumplimiento $(D D)$ se calcula como

$$
D D=\frac{V_{A}-D P}{\sigma_{A} V_{A}}
$$

Donde, $V_{A}$ representa el valor de los activos y $\sigma_{A}$ su volatilidad. Para una empresa con responsabilidad limitada, el patrimonio el cual está determinado por el valor de mercado de las acciones $\left(V_{E}\right)$, tiene el derecho residual sobre los activos después de que se hayan cumplido todas las demás obligaciones. De esta forma, una opción de compra sobre los activos con precio de ejercicio igual a la deuda tiene las mismas propiedades indicadas en el modelo de Merton. Así, al tomar los mismos supuestos sobre $V_{A}, V_{E}$ y $V_{D}$, dados por las ecuaciones 1 y $5, D D$ se podría estimar usando la ecuación 6 b. Entonces se tiene

$$
D D=\frac{\ln \left(\frac{V_{A}}{V_{D}}\right)+\left(r-\frac{1}{2} \sigma_{A}^{2}\right) T}{\sigma_{A} \sqrt{T}}
$$

Sin embargo, como $\sigma_{A}$ no es observable, $D D$ se aproxima mediante la ecuación $8 \mathrm{~b}$, mientras que la probabilidad de incumplimiento (riesgo-neutral) en un vencimiento $T$, está determinada por

$$
P\left[V_{A} \leq V_{D}\right]=N(-D D)
$$


Como resultado, este modelo establece que $D D$ es suficiente para estimar la probabilidad de incumplimiento, donde el numerador de la ecuación $8 \mathrm{~b}$ determina el nivel de apalancamiento financiero, el cual está asociado al nivel de riesgo financiero que tiene la empresa, mientras que el denominador refleja su nivel de riesgo de mercado.

\section{Modelo estimación del riesgo de crédito en proyectos de infraestructura}

La estimación de la probabilidad de incumplimiento, junto con lo demás componentes requeridos para la cuantificación del riesgo de crédito (pérdida esperada) en el sector corporativo, han sido ampliamente estudiados en la teoría financiera, donde la evaluación crediticia se realiza a partir de la información que proveen las hojas de balance y los estados financiaros, así como su desempeño histórico (Gatti et al., 2007). En este contexto, los modelos estructurales presentan ventajas para su estimación, sin embargo, en los proyectos de infraestructura el desarrollo de modelos propios ha sido escaso y los modelos presentados atrás presentan limitaciones. Al respecto, el Comité de Basilea en el marco del acuerdo de Basilea II (Basilea, 2004) resaltó las limitaciones del uso de modelos de riego de crédito convencionales ${ }^{6}$ en el financiamiento estructurado de proyectos de infraestructura. A partir de la distinción entre el financiamiento corporativo y el financiamiento estructurado de proyectos, el Comité recomendó la implementación de metodologías diferentes para determinar la pérdida esperada del riesgo de crédito (EL, por su sigla en inglés), teniendo en cuenta los tres componentes:

$$
E L=D P \times L G D \times E A D
$$

Donde, $D P$ es la probabilidad de incumplimiento, $L G D$ es la pérdida dado el incumplimiento y, $E A D$ la exposición al incumplimiento.

Como se indicó atrás, en la financiación de proyectos, el flujo de efectivo es el factor determinante del valor de los activos y, por tanto, determina la capacidad de pago de la deuda. Inicialmente, este valor es el único referente ${ }^{7}$ que se tiene en cuenta en la estructuración financiera del proyecto para determinar el tamaño de la deuda. Por tanto, el riesgo de crédito está asociado a la incertidumbre futura de los flujos de efectivo del proyecto $\mathrm{y}$, su dinámica determina la capacidad de pago y la probabilidad de entrar en incumplimiento. Así, la evaluación del riesgo de crédito está determinado por la posibilidad de que, en un momento dado, los flujos de efectivo sean insuficientes para pagar el servicio de deuda.

\footnotetext{
${ }^{6}$ Se hace esta distinción para referirse a los modelos de Merton y KMV de Moody's.

${ }^{7}$ Según Gatti (2008), generalmente el proyecto posee activos que definen un carácter específico determinado por su diseño contractual
} 
Trabajos recientes han demostrado que los modelos estructurales pueden utilizarse para estimar el riesgo de crédito en proyectos (ver Blanc-Brude, et al., 2014; Blanc-Brude y Hasan, 2016), sin embargo, estos modelos ameritan un tratamiento especial con algunos ajustes. Por ejemplo, la incorporación de derechos de control para los prestamistas (Borgonovo y Gatti, 2013), así como covenants de deuda incorporan un efecto importante en la estimación de la pérdida esperada y sus componentes, requiriendo así una redefinición del incumplimiento. De igual forma, la especificación del modelo en términos del flujo de efectivo permite utilizar información observable para obtener los parámetros del modelo. Por ejemplo, Blanc-Brude y Hasan (2016) demostraron que al comprender la dinámica de la ratio de cubertura del servicio de deuda ( $D S C R$, por sus siglas en inglés), junto con el perfil de amortización y las garantías disponibles ${ }^{8}$, las cuales son observables, es suficiente para implementar un modelo estructural.

El primer paso clave para estimar el riesgo de crédito, corresponde a la modelación de los flujos de efectivo para cada momento t, donde el incumplimiento se da en aquellos escenarios cuando este es insuficiente para pagar el servicio de deuda (capital e intereses). Así, la determinación de la capacidad de pago del proyecto a partir de $D S C R$ es fundamental para su aplicación. $D S C R$ mide la cantidad de efectivo disponible para realizar el pago del servicio de la deuda (CFADS, por su sigla en inglés) en un momento t, y se estima como

$$
D S C R_{t}=C F A D S_{t} / D S_{t}
$$

Donde, $D S_{t}$ representa el servicio de deuda en $t$. Cuanto más alto sea $D S C R$, más efectivo disponible tendrá el proyecto para cumplir con sus obligaciones de deuda. La dinámica de $D S C R$ facilita la implementación de un modelo estructural de riesgo de crédito, ya que los niveles de DSCR por definición determinan los umbrales de incumplimiento. Según los autores, el $D S C R$ da una definición inequívoca del punto de
i. $\quad$ incumplimiento "estricto", determinado por $D S C R=1$;
ii. incumplimiento "técnico", determinado por $D S C R=1, x$.

De esta forma, el conocimiento de la dinámica de $D S C R$ es suficiente para estimar la distancia al incumplimiento $(D D)$. De igual forma, la dinámica de $D S C R$ también se puede combinar con el servicio de deuda futuro para calcular el valor esperado y la vola-

\footnotetext{
${ }^{8}$ Una forma de garantía para los prestamistas es aquella que está disponible en la "cola" de la vida de la deuda. Según, Blanc-Brude y Hasan (2016), la presencia de estas características distintivas hace que los modelos estructurales de riesgo de crédito sean una opción natural.
} 
tilidad del flujo de efectivo futuro. A pesar de su estimación directa, tanto de $D D$ como de la probabilidad de incumplimiento, los demás componentes de la $E L$ pueden tener limitaciones que se deben señalar. Por lo general, los prestamistas, en su esfuerzo de reducir su exposición al riesgo, exigen al SPV la creación de cuentas de reserva de efectivo para cubrir parte del servicio de deuda futuro, lo cual aumenta su tasa de recuperación y reduce la exposición total en caso de un evento de incumplimiento. Por lo tanto, la estimación de estos componentes requiere un análisis por etapas, como se discutirá más adelante.

\section{Dinámica de DSCR y determinación de la probabilidad de incumplimiento}

Dado que el comportamiento del $D S C R$ está fuertemente relacionado con el riesgo de crédito del proyecto, el conocimiento de la distribución del $D S C R$ es suficiente para su estimación. Si se asume que la dinámica estocástica del DSCR sigue un proceso log-normal, se tiene

$$
d D S C R_{t} / D S C R_{t}=\mu d t+\sigma d W_{t}
$$

Donde $\mu$ y $\sigma$ representan la tasa de deriva del $D S C R$ y su correspondiente volatilidad, las cuales son conocidas y constantes, mientras que $W_{t \in[0, T]}$ es un proceso estándar de Wiener. Ahora, como el punto de incumplimiento (técnico) está determinado por

$$
D S C R_{t} \equiv C F A D S_{t} / D S_{t}<1 . x
$$

Donde, CFADS es el flujo del efectivo disponible para el servicio de la deuda, entonces de forma análoga al modelo KMV de Moody's, la distancia al incumplimiento en cada t es

$$
D D_{t}=\frac{C F A D S_{t}-D S_{t}}{\sigma_{C F A D S} C F A D S_{t}}
$$

Donde $\sigma_{C F A D S}$ es la volatilidad de los CFADS. Con el propósito de evitar cualquier problema debido a la dependencia de escala de CFADS, el flujo de efectivo se re-expresa en términos de $D S C R$

$$
C F A D S_{t}=D S C R_{t} \times D S_{t}
$$

Al usar esta definición de CFADS, la distancia al incumplimiento se puede expresar como

$$
D D_{t}=\frac{1}{\sigma_{C F A D S}}\left(1-\frac{1}{D S C R_{t}}\right)
$$


De igual forma, la ecuación 16 se puede reescribir como una función exclusiva del $D S C R$ al expresar la volatilidad del $C F A D S$ en función de la volatilidad del $D S C R\left(\sigma_{D S C R}\right)$

$$
D D_{t}=\frac{1}{\sigma_{D S C R}} \frac{D S_{t-1}}{D S_{t}}\left(1-\frac{1}{D S C R_{t}}\right)
$$

Donde, $\sigma_{C F A D S}=\left(\frac{D S_{t-1}}{D S_{t}}\right) \sigma_{D S C R}{ }^{9}$. De igual forma, la probabilidad de incumplimiento $(P(t, T))$ viene determinada por $D D_{t}$ :

$$
P(t, T)=N\left(-D D_{t}\right)
$$

Sin embargo, esta medida incorpora las preferencias de riesgo de los inversionistas. Dado que la ecuación 12 muestra la dinámica del $D S C R$ en términos de la tasa de deriva $(\mu)$, la estimación de la probabilidad requiere un ajuste riesgo-neutral, donde se introduce la prima por riesgo (coeficiente de Sharpe $\lambda$ ), el cual es estimado para un horizonte $T: \lambda=\frac{\mu-r}{\sigma} \sqrt{T^{10}}{ }^{10}$. De esta forma se tiene:

$$
Q(t, T)=N\left(N^{-1}[P(t, T)+\lambda)\right.
$$

Donde, $Q(t, T)$ es la probabilidad de incumplimiento riesgo-neutral. De igual forma, la estimación de la probabilidad de incumplimiento también se puede realizar en un contexto de simulación de Monte Carlo, donde se generan trayectorias posibles de los $D S C R_{t}$ y/o $C F A D S_{t}$, buscando reflejar el mayor número de trayectorias posibles. Por tanto, al modelar los flujos de efectivo es posible reflejar si se puede generar un evento de incumplimiento durante la vida del proyecto.

La ventaja de este enfoque de simulación se debe a su capacidad práctica para incorporar las distintas fuentes de riesgo del proyecto, asociados a incertidumbres tanto operativas como de mercado mediante supuestos probabilísticos que reflejen su propia naturaleza ${ }^{11}$, así como sus correlaciones. De esta forma, la identificación de los factores de riesgo del proyecto y su modelación puede tener ventajas prácticas. A continuación, se detalla la implementación del modelo de simulación de Monte Carlo por etapas.

La primera etapa de la implementación del modelo de Monte Carlo, como muestra la figura 1, comprende la incorporación de las incertidumbres al modelo financiero (el flujo

\footnotetext{
${ }^{9}$ Cabe resaltar que sí, dadas las condiciones del crédito, se adopta un esquema de amortización con cuota fija, entonces: $\sigma_{C F A D S}=\sigma_{D S C R}$, dado que $D S_{t-1}=D S_{t}$, para todo el periodo.

${ }^{10}$ Este ajuste riesgo-neutral tiene como objetivo incorporar las preferencias de riesgo de los inversionistas en la modelación del CFADS. Lo anterior indica que, si los flujos de efectivo reflejan la naturaleza riesgosa de la inversión, estos deben ser descontados a la tasa libre de riesgo en el modelo de valoración.

${ }^{11}$ La ventaja de adoptar diferentes tipos de funciones de probabilidad se debe a que los parámetros del modelo se pueden obtener a partir de la evidencia empírica.
} 
de caja), mediante la asignación de supuestos probabilísticos en las diferentes fuentes de incertidumbre del proyecto. Como resultado de este proceso se obtiene una función de distribución de probabilidad que caracteriza al valor presente de los flujos de efectivo del proyecto. La tasa de variación del valor presente de los flujos de efectivo comprende el principal insumo para la estimación de la volatilidad del $\operatorname{DSCR}\left(\sigma_{D S C R}\right)$.

La segunda etapa de la implementación del modelo de Monte Carlo (ver figura 3) comprende la simulación de trayectorias del $D S C R_{t}$, donde se asume que este sigue un proceso estocástico del tipo mBg. Luego, para cada momento t se estima la probabilidad de incumplimiento, por ejemplo, que se encuentre por debajo de un umbral definido en $1, x$. El punto de incumplimiento se define a partir de los covenants que se imponen en el contrato de la deuda.

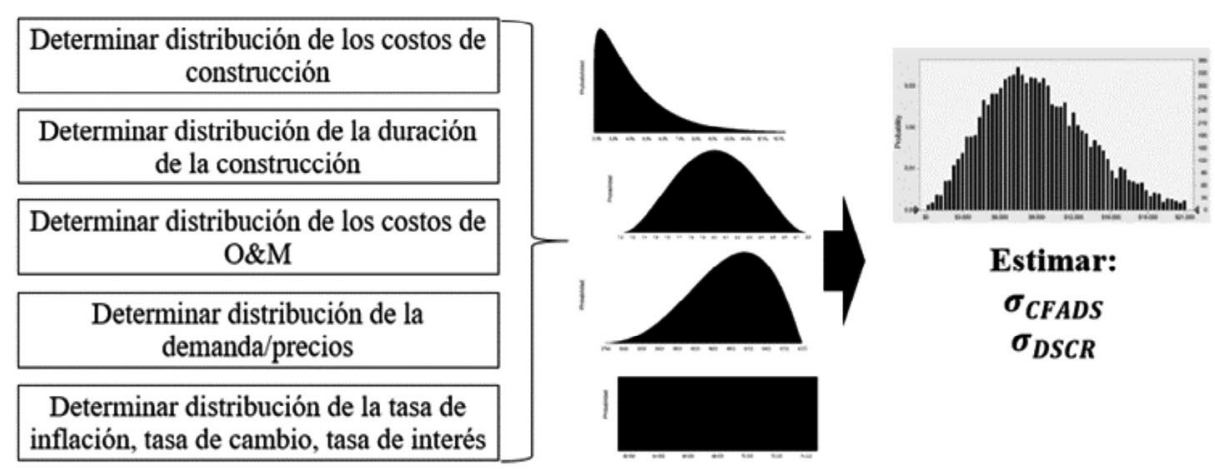

Figura 2. Etapa 1: Modelación de las incertidumbres y estimación de la volatilidad Fuente: elaboración propia

\section{DSCR}

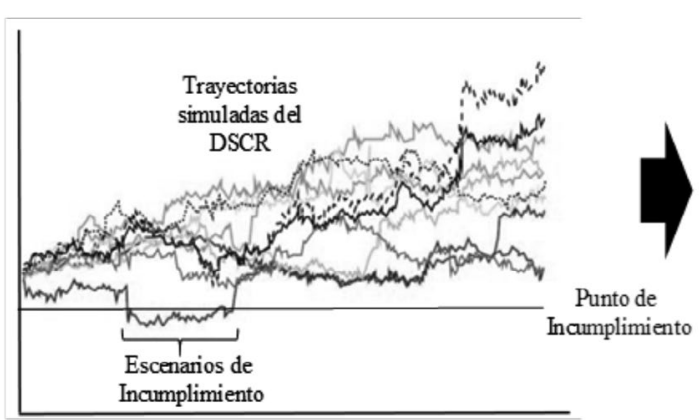

Tiempo
Para cada $t$

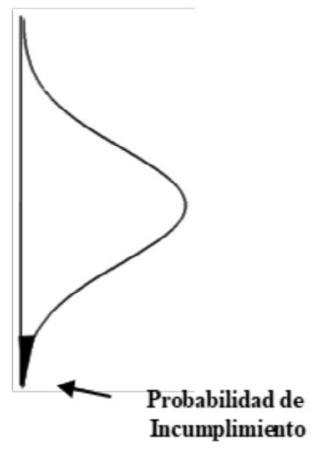

Figura 3. Etapa 2: Simulación de trayectorias del DSCR y estimación de la probabilidad de Incumplimiento Fuente: elaboración propia 
Además, este tipo de aplicaciones con la técnica de simulación de Monte Carlo es utilizada por Gatti et al. (2007) para la estimación del valor en riesgo (VaR, por su sigla en inglés) del proyecto. El lector debe tener presente que la modelación de factores de riesgo representa un tema crítico, donde los juicios y criterios propios tienen un gran efecto en la evaluación de riesgo y, por ende, en la estimación de la probabilidad de incumplimiento.

\section{Reestructuración del acuerdo de financiación y análisis del incumplimiento}

Hasta ahora, el riesgo de crédito se ha analizado en un contexto donde la probabilidad de incumplimiento se determina a partir de la capacidad de pago del proyecto. Sin embargo, el análisis anterior debe extenderse para incorporar el efecto de una reestructuración del contrato de deuda en caso de un evento de incumplimiento. Dado que los prestamistas pueden aceptar una reestructuración, en lugar de permitir que el proyecto incumpla el pago de la deuda dadas las consecuencias adversas que esto produce como la pérdida de calidad crediticia y el aumento de los costos de financiación.

Según Gatti (2008), el contrato de deuda puede incorporar cláusulas que les permite a los prestamistas tomar medidas de control para evitar que la capacidad pago del proyecto se vea afectada. Por tanto, en un evento de incumplimiento, los prestamistas estarán dispuestos a tomar medidas correctivas para mantener el proyecto en normal funcionamiento ${ }^{12}$, medidas que incluso pueden generar una pérdida para ellos, por ejemplo, cuando la reestructuración extiende los plazos de amortización, reduce el pago de intereses o, por el contrario, acelera su tasa de recuperación. Por tal razón, los componentes del riesgo de crédito como $L G D$ y $E A D$ pueden cambiar en un escenario de incumplimiento, por lo que los modelos de estimación deben incorporar estos ajustes.

Aunque su aplicación puede ser compleja, estos se pueden incorporar a partir de: i) la descomposición de Black-Cox partiendo del modelo estructural; y ii) simulación de Monte Carlo por etapas. A continuación, se detallan estas propuestas.

\section{Descomposición de Black-Cox}

La descomposición Black-Cox, propuesta por Black y Cox (1976), comprende un método utilizado para valorar deuda corporativa en escenarios de reestructuración o refinanciación cuando el valor de los activos de la empresa alcanza un límite (inferior o superior). De esta

\footnotetext{
${ }^{12}$ Es común que estas medidas correctivas les permita renegociar las condiciones actuales de la deuda, cambiando los plazos de amortización, el pago de intereses, la incorporación de créditos subordinada.
} 
forma, el modelo propuesto por Blanc-Brude y Hasan (2016) debe requerir de un ajuste adicional, dado que solo contempla la dinámica que proporciona el flujo de efectivo. Este ajuste se presenta a partir de una descomposición que incorpora cuatro funciones de pago que se presentan a continuación.

1. $\quad P\left(T_{D}, C F A D S_{T_{D}}\right)$ : pago final al vencimiento de la deuda, donde T_D representa el vencimiento de la deuda.

2. $\underline{P}\left(\tau, \underline{C F A D S_{\tau}}\right)$ : valor de los activos si el CFADS alcanza el límite inferior, es decir un incumplimiento, en el tiempo $\tau$, llevando a un estado de reestructuración.

3. $\bar{P}\left(\tau, \overline{C F A D S_{\tau}}\right)$ : valor de los activos si el CFADS alcanza el límite superior en el tiempo $\tau$ y corresponde a un estado de refinanciación, debido a que permite acelerar el pago de la deuda, junto con una reducción en sus costos.

4. $p^{\prime}\left(t, C F A D S_{t}\right)$ : pago de la deuda realizado antes del vencimiento o la reestructuración.

Así, el valor total de los activos corresponde al valor presente (esperado) de la suma de las cuatro funciones de pago, bajo la medida de probabilidad riesgo-neutral. Si se define $h\left(V_{t}, t\right)$ como el valor de los activos en un momento t, $K(\cdot)$ denota el intervalo $\underline{C F A D S_{\tau}}(\cdot)$,

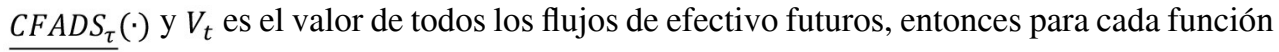
de pagos se tiene:

$$
\begin{aligned}
& h_{\mathbf{1}}\left(V_{t}, t\right)=E\left[e^{-r_{T_{D, t}}\left(T_{D}-t\right)} P\left(T_{D}, C F A D S_{T_{D}}\right)\right] \\
& =e^{-r_{T_{D, t}\left(T_{D}-t\right)}} \int_{k(T)}\left(T_{D}, C F A D S_{T_{D}}\right) d F^{*} \\
& h_{2}\left(V_{t}, t\right)=\int_{t}^{T} e^{-r_{\underline{C F A D S}, t}\left(\underline{T}_{\underline{\underline{C F A D S}}}-t\right)} \times \underline{P}\left(\operatorname{CFADS}_{T_{\underline{\underline{C F A D S}}}} T_{\underline{C F A D S}}\right) d F_{T_{\underline{\underline{C F A D S}}}^{*}}
\end{aligned}
$$

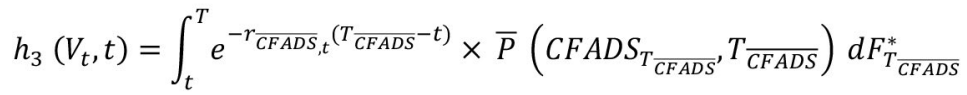

$$
\begin{aligned}
& h_{4}\left(V_{t}, t\right)=\int_{t}^{T_{D}} e^{-r_{s, t}(s-t)} \times\left[\int_{k(T)} p^{\prime}\left(C F A D S_{s}, s\right) d F^{*}\left(C F A D S_{s}, s\right)\right] d s
\end{aligned}
$$

Donde, $d F^{*}$ es la probabilidad de que $C F A D S$ descienda del umbral durante $T_{D}, F_{T_{C F A D S}}^{*}$ define la función de densidad cuando el CFADS alcanza el límite de incumplimiento, mientras 
$F_{T_{\overline{C F A D S}}}^{*}$ define la función de densidad alcanza el límite superior (refinanciación).

De esta forma, el valor total de los activos $V^{s}\left(V_{t}, t\right)$ será determinado por

$$
V^{S}\left(V_{t}, t\right)=\sum_{i=1}^{i=4} h_{i}\left(V_{t}, t\right)
$$

Donde $h_{i}\left(V_{t}, t\right)$ es el valor determinado por la i-ésima función de pagos.

Simulación por etapas basado en la técnica de simulación de Monte Carlo

La incorporación de eventos de reestructuración en la simulación requiere primero determinar, como en caso de un incumplimiento, los factores desencadenantes afectan los componentes de $L G D$ y $E A D$. Con respecto a la $L G D$, se sabe que la tasa de recuperación depende claramente de los CFADS futuros y, por tanto, del valor del proyecto en caso de incumplimiento. Siguiendo a Gatti (2008), se debe incorporar un tercer nivel (etapa 3) al proceso indicado en la sección anterior. Una vez se obtengan los resultados del modelo de Monte Carlo (etapas 1 y 2), se debe ejecutar un conjunto de simulaciones para cada escenario por incumplimiento con el objetivo de obtener la distribución de los valores de $L G D$. Además, este ejercicio puede proveer información adicional relevante para el análisis de riesgos del proyecto, como lo es el VaR definido a un nivel de confianza (por ejemplo, 99\%) y un horizonte de tiempo determinado, o para cada año de vida de la deuda.

\section{Aplicación en un proyecto de concesión vial}

El modelo estructural de riesgo de crédito presentado será implementado en un proyecto hipotético de concesión de una carretera de peaje en Colombia, el cual está estructurado bajo un esquema BOMT (build-operate-maintenance-transfer). El objetivo de esta aplicación es ilustrar al lector en la implementación paso a paso del modelo propuesto, aunque no se dará un abordaje completo de este.

\section{Información del proyecto y supuestos}

El proyecto comprende una concesión de carretera de peaje con extensión de $120 \mathrm{~km}$ por un periodo de 20 años, de los cuales los tres primeros corresponden a la fase de financiación y construcción y los 17 restantes a la fase de operación y mantenimiento, periodo en el cual el 
SPV tendrá a su cargo la administración de 2 casetas de peaje. La inversión total estimada del proyecto asciende a $\$ 716.750$ millones de pesos (COP), de los cuales los inversionistas privados aportaran el $40 \%$, mientras el $60 \%$ restante se obtiene con bancos locales a través de un crédito sindicado. En este crédito sindicado se asume una duración de 12 años para la amortización con un periodo de gracia de 3 años (fase de construcción). Además, en la amortización se asume un método de cuota fija (anualidad) con una tasa de interés del $8 \%$ y, se considera como principal covenant de la financiación una ratio de cobertura () mínimo de 1,2. La tabla 1 resume esta información y los demás supuestos del modelo financiero del proyecto.

A partir de esta información, se estiman el CFADS y el DSCR para cada año del proyecto, teniendo en cuenta que el monto de servicio de deuda bajo la modalidad indicada es de $\$ 57.065$ millones (COP) para el periodo de amortización (años 4 a 15) ${ }^{13}$. Las figuras 4a y $4 \mathrm{~b}$ presenta estos resultados.

\section{Tabla 1}

Supuestos del proyecto

\begin{tabular}{ll}
\hline Duración & 20 años \\
\hline Moneda & Peso COP \\
Inflación anual esperada & $3 \%$ \\
Inversión total (millones) & $\$ 716.750$ \\
Costos de operación (millones) & $\$ 5.700$ \\
Tarifa de peaje (promedio) & $\$ 19.340$ \\
Trafico promedio diario (TPD) & 7.275 \\
Crecimiento anual del TPD & $4,5 \%$ \\
Impuesto a la renta & $34 \%$ \\
Equity (\%) & $40 \%$ \\
Deuda (\%) & $60 \%$ \\
Tasa de interés de la deuda & $8 \%$ \\
DSCR mínimo & 1,2 \\
Costo del equity & $10 \%$ \\
Tasa libre de riesgo & $4 \%$ \\
\hline Fuente: elaboración propia &
\end{tabular}

Fuente: elaboración propia

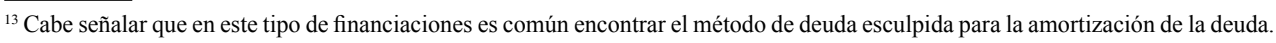
Aunque este método ofrece ventajas notorias para implementar un modelo multietapa en la simulación de Monte Carlo, no se va a usar en esta aplicación.
} 


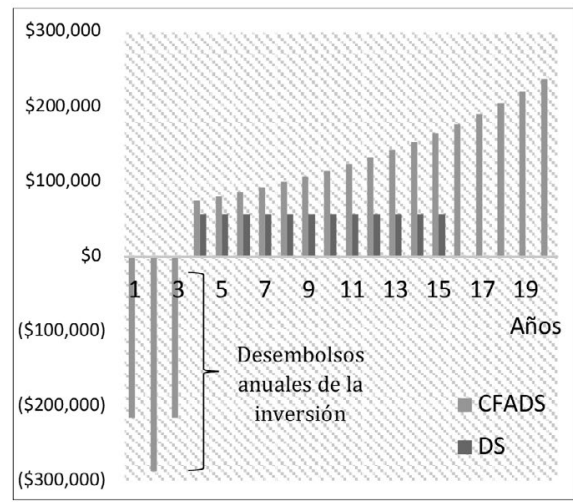

(a) Proyección de los flujos de efectivo (CFADS) y del servicio de deuda (DS)

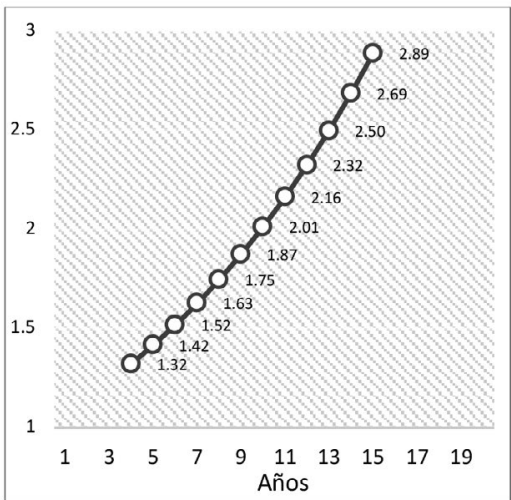

(b) Ratio de cobertura de la deuda (DSCR)

Figura 4. Estimación de la distancia al incumplimiento y la probabilidad de incumplimiento Fuente: elaboración propia

Con estos resultados, se encuentra que el proyecto cumple con la capacidad de pago de la deuda, al encontrar un DSCR mínimo de 1,32 para el periodo de amortización. Sin embargo, este resultado parte de una representación determinística del modelo financiero y no refleja completamente el riesgo asumido. Por tanto, este análisis debe ser complementado.

\section{Estimación de la distancia al incumplimiento y la probabilidad de incumplimiento}

El primer paso para la estimación de la probabilidad de incumplimiento corresponde a la estimación de la volatilidad de los CFADS ( $\sigma_{C F A D S}$ ) y/o de los DSCR ( $\left.\sigma_{C F A D S}\right)$. Cabe resaltar que, sin importar cual se utilice, bajo el esquema de amortización de cuota fija asumido se tiene que: $\sigma_{D S C R}=\sigma_{C F A D S}$. Dada la dificultad que se presenta para la estimación de este parámetro, se propone adoptar un enfoque similar al utilizado en la teoría de opciones reales ${ }^{14}$. Para ello, se utiliza el método desarrollado por Brandão, Dyer y Hahn (2012). Bajo este modelo se obtiene la volatilidad del valor presente de los CFADS utilizando la técnica de simulación de Monte Carlo ${ }^{15}$.

\footnotetext{
${ }^{14}$ Cabe resaltar que no es la primera vez que se utiliza este enfoque para abordar problemas propios de la financiación de proyectos de infraestructura. La teoría de opciones reales ha sido incorporada para el análisis y valoración de riesgos, donde se asume que el valor del proyecto sigue un proceso de difusión del tipo $\mathrm{mBg}$.

${ }^{15}$ Además, al utilizar este enfoque de simulación se identifican dos ventajas considerables: i) al incorporar las distintas fuentes de riesgo (tanto operativas como de mercado) mediante supuestos probabilísticos se puede estimar su efecto agregado sobre el proyecto en una única medida; ii) estos supuestos probabilísticos determinan el escenario base de simulación, sobre el cual se puede estimar el componente de LGD o la tasa de recuperación en escenarios de incumplimiento, el cual representa un insumo necesario para la estimación de la pérdida esperada.
} 
La tabla 2 presenta los supuestos probabilísticos que se utilizaron para la modelación de las diferentes fuentes de riesgo del proyecto y estimación del parámetro de volatilidad.

Tabla 2

Supuestos probabilísticos del modelo financiero

\begin{tabular}{llll}
\hline Variable & Función de probabilidad & $\begin{array}{l}\text { Parámetros } \\
\text { Media }\end{array}$ & Desv. Estándar \\
\hline TPD & Log-normal & 6.100 & 540 \\
Costos de operación (millones) & Log-normal & $\$ 5.700$ & $\$ 500$ \\
Crecimiento anual del TPD & Normal & $2.0 \%$ & $0,2 \%$ \\
Mantenimiento & Normal & $4.5 \%$ & $0,45 \%$ \\
Inflación & Normal & $3.0 \%$ & $0,3 \%$ \\
\hline
\end{tabular}

Fuente: elaboración propia

Estos supuestos probabilísticos en las variables identificadas se incorporan de tal forma que sean consistentes con el proceso de difusión que se asume en el $D S C R$, como se indica en la ecuación 12. En el ejercicio de simulación se emplean 100.000 iteraciones usando Crystall Ball. Como resultado, se obtiene una volatilidad del $18 \%$. Una vez se tiene la volatilidad se procede a estimar la prima por riesgo $(\lambda)$, la cual con una tasa libre de riesgo de $4 \%$ es 0.0278 . Una ventaja de este enfoque, como indica Gatti (2008), corresponde a la representación de las principales fuentes de riesgo del proyecto.

El siguiente paso corresponde a la estimación de la distancia al incumplimiento $(D D)$ a cada año del periodo de amortización. De igual forma, se define el punto de incumplimiento en sus dos versiones (estricto y técnico) correspondientes a $D S C R_{t}<1$ y $D S C R_{t}<1.2$, respectivamente. Las figuras $5 \mathrm{a}$ y $5 \mathrm{~b}$ presentan las estimaciones de la distancia al incumplimiento y la probabilidad de incumplimiento.

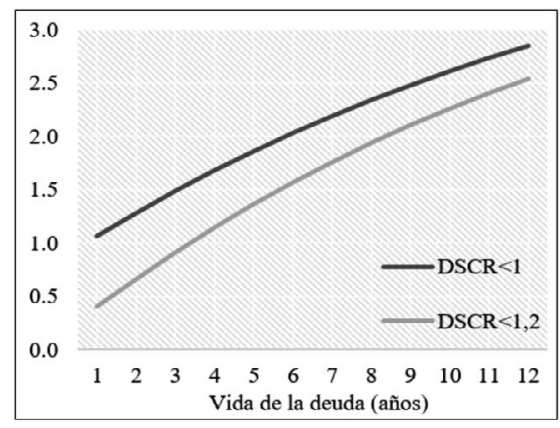

a. Distancia al incumplimiento

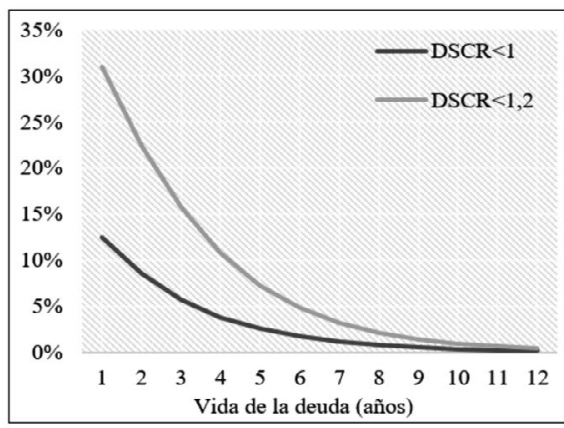

b.Probabilidad de incumplimiento

Figura 5. Estimación de la distancia al incumplimiento y la probabilidad de incumplimiento Fuente: elaboración propia 
En su primer año, el proyecto alcanza el $D S C R$ más bajo de todo el periodo $(1,32)$, por lo tanto, la probabilidad de incumplimiento en este año va a ser la más alta. A medida que mejora su capacidad de pago, es decir, aumenta la relación entre $C F A D S$ y el servicio de deuda para cada t, la probabilidad va descendiendo rápidamente, la cual se identifica para los dos puntos de incumplimiento.

\section{Estimación de la pérdida esperada y el valor en riesgo (VaR) crediticio}

EL toma una dinámica similar a la probabilidad incumplimiento a lo largo del periodo de amortización, dada su relación directa con esta y la forma como evolucionan EAD y LGD, como se muestran las figuras $6 \mathrm{a}$ y $6 \mathrm{~b}$, diferenciando las estimaciones para el incumplimiento técnico y el estricto. El hecho de que esta probabilidad descienda a niveles casi nulos al final del periodo indica que $E L$ desciende a cero. Particularmente, desde el año 9 de la vida de la deuda es casi cero. En otras palabras, sin tener en cuenta los otros dos componentes, el comportamiento asintótico que toma la probabilidad se refleja directamente en $E L$.

Lo mismo sucede con los componentes $E A D$ y $L G D$ (figura 6a): al final del periodo, la deuda se ha pagado casi completamente, por tanto, estos dos se acercan a cero al igual que la probabilidad y, por tanto, la $E L$ es casi nula. Esta dinámica, tiene una utilidad enorme para el análisis de riesgo de crédito y las provisiones de los bancos que participaron en su financiación, al permitir una estimación directa y sencilla de estos. Además, partiendo de esta información se obtiene el valor presente de la pérdida esperada total es cada caso: \$65.550 millones en caso de incumplimiento técnico y \$221.900 millones en incumplimiento estricto. Para obtener estos resultados se descuenta cada $E L$ anual a la tasa de interés del crédito (8\%).

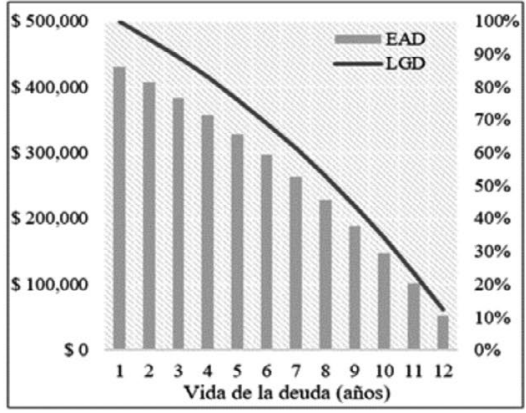

(a) Estimación de EAD y LGD (eje secundario)

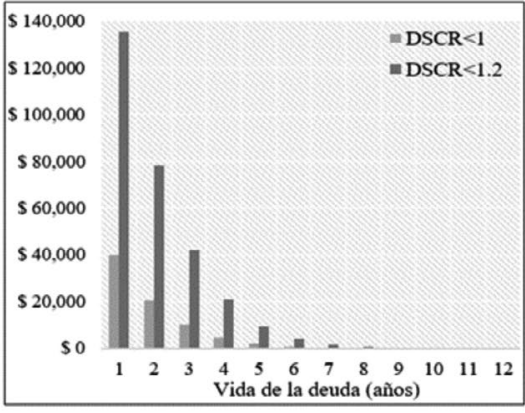

(b) Estimación de EL

Figura 6. Estimación de la perdida esperada

Fuente: elaboración propia 
Por ahora, solo se ha hecho una estimación estática de la EL. Como se indicó en la sección anterior, se pueden incorporar niveles adicionales o etapas en la simulación de Monte Carlo, donde se obtiene la función de distribución de la EL. Una vez más con ayuda de Crystal Ball se obtienen las funciones de distribución. La figura 7 presenta la función de densidad del valor presente (VP) de $E L$ teniendo en cuenta los dos tipos de incumplimiento, así como el VaR crediticio (al 99\%), es decir la máxima perdida probable de los bancos que participaron en la financiación del proyecto.

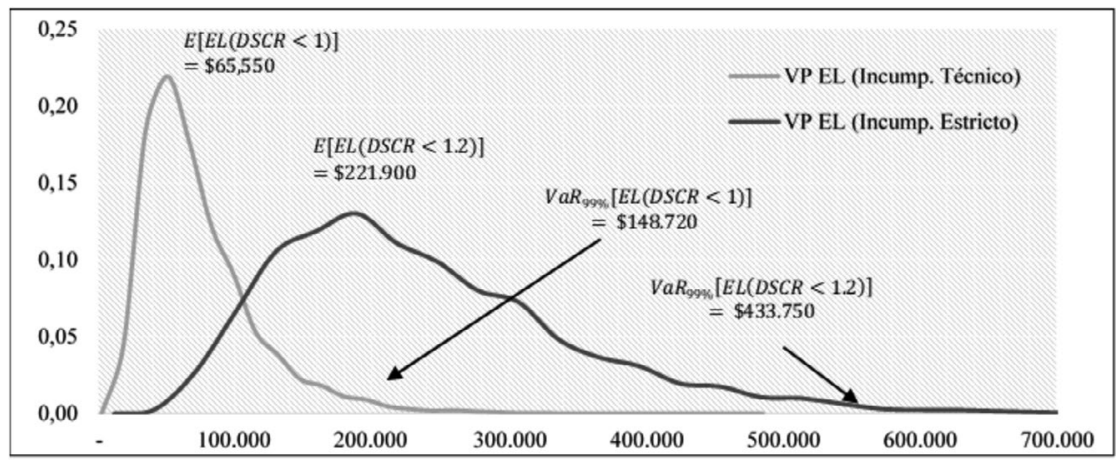

Figura 7. Estimación probabilística de la pérdida esperada y el valor en riesgo (VaR) crediticio Fuente: elaboración propia

Cabe resaltar que, por simplicidad, en las estimaciones de $E L$ no se consideran las reservas de deuda. Lo anterior, tiene aún más relevancia dado que la estimación depende directamente de las condiciones impuestas por los bancos y, que se ven reflejadas en los covenants. En la medida que los bancos asuman un mayor riesgo, mayor van a ser las exigencias en materia de requerimientos como reservas de deuda.

Análisis probabilístico de la tasa de recuperación $(R R)$

Para finalizar el análisis del enfoque de simulación de Monte Carlo, se realizará un análisis probabilístico de la tasa de recuperación (RR), teniendo en cuenta la dinámica del DSCR como indican las ecuaciones 12 y 13. Para ello, se tomara como punto de referencia solo el incumplimiento estricto, es decir $D S C R_{t}<1$, donde al igual que Blanc-Brude y Hasan (2017), se asume que en un escenario de incumplimiento estricto se liquidará el SPV. Aquí los bancos podrán recuperar el monto de deuda pagada por el SPV hasta la fecha t. Este análisis se puede 
realizar tomando o no las reservas de deuda acumuladas hasta ese momento, las cuales se han ignorado por ahora.

La figura 8 presenta los resultados de la simulación. Como se observa allí, la estimación de la tasa de recuperación esperada $(E[R R])$ difiere un poco, aunque esta diferencia no es importante.

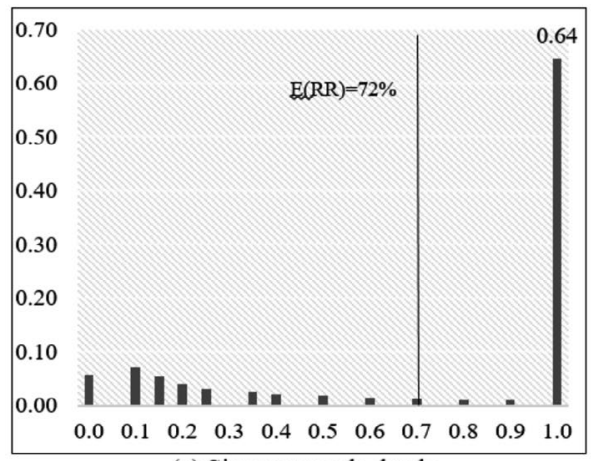

(a) Sin reservas de deuda

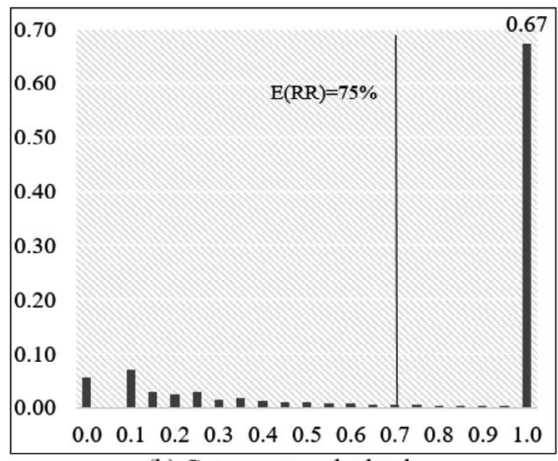

(b) Con reservas de deuda

Figura 8. Estimación de la tasa de recuperación (RR)

Fuente: elaboración propia

La incorporación de las reservas de deuda aumenta el valor de $E[R R]$ de $72 \%$ a $75 \%$, mientras que el número de escenarios en los que los bancos alcanzan una recuperación completa de la deuda pasa de un 64\% a un 67\%. Los resultados anteriores concuerdan con los análisis realizados por Moody's (2017) y Jobst (2018), en los cuales se encuentra que la tasa de recuperación para el sector de infraestructura a nivel mundial (en promedio) está en el rango 70\%-80\%.

\section{Conclusiones}

Este trabajo presentó un modelo estructural de estimación del riesgo de crédito mediante un enfoque integrado que combina la dinámica de la capacidad de pago de la deuda siguiendo los desarrollos propuestos por Blanc-Brude y Hasan (2016), junto con la técnica de simulación de Monte Carlo. Una ventaja importante de este enfoque es que permite una estimación directa de todos los componentes de la pérdida esperada (EL), como lo son la probabilidad de incumplimiento (DP), la exposición (EAD) y la pérdida dado el incumplimiento (LGD), o la tasa de recuperación (RR), teniendo en cuenta la incertidumbre futura del flujo de efectivo 
del proyecto disponible para el pago del servicio de deuda y su efecto en la pérdida potencial. Además, el modelo propuesto se puede implementar sin mayor dificultad hasta lograr una estimación probabilística de todos los componentes.

De esta forma, se ofrece a los bancos una herramienta útil para cuantificar el riesgo de crédito en proyectos de infraestructura. Cabe anotar que, dada la naturaleza compleja de un evento de incumplimiento en estos proyectos, el tratamiento del riesgo de crédito está fundamentado en los umbrales determinados por el DSCR, los cuales explican la ocurrencia de un incumplimiento "estricto" o "técnico", este último justificado por la presencia de los covenants y derechos de control significativos para los bancos.

De igual forma, aún se presentan algunas limitaciones del modelo propuesto que deben seguir indagando en aras de presentar un modelo mucho más robusto y completo. Primero, la fiabilidad de las estimaciones se basa en las proyecciones de los flujos de efectivo (CFADS) e incorpora un componente subjetivo que está limitado a la experiencia y el conocimiento del estructurador de la iniciativa y puede generar algunos sesgos. Segundo, un análisis adecuado de los escenarios de refinanciación o reestructuración del crédito, los cuales permitirían identificar el cronograma óptimo de amortización de tal forma que se maximice la tasa de recuperación (o se minimice el riesgo) para los bancos, requeriría la incorporación de la teoría de opciones reales como sugieren Blanc-Brude, Hasan y Whittaker (2016).

\section{Referencias}

Aragones, J., Blanco, C., e Iniesta, F. (2009). Modelizacion del riesgo de credito en proyectos de infraestructuras. Innovar, 19(35), 65-80. Disponible en https://www.redalyc.org/ pdf/818/81819026006.pdf. Consultado el 8 de diciembre de 2018.

Basel Committee on Banking Supervision (2004). Basel II: International Convergence of Capital Measurement and Capital Standards: a Revised Framework. Bank for International Settlements, Basel. Disponible en https:// www.bis.org/publ/bcbs128.htm. Consultado el 10 de enero de 2019.

Boussabaine, A. (2013). Risk pricing strategies for public-private partnership projects. John Wiley \& Sons.

Black, F. y Cox, J. (1976). Valuing corporate securities: Some effects of bond indenture provisions. The Journal of Finance, 31(2), 351-367. https://doi.org/10.1111/j.1540-6261.1976.tb01891.x

Black, F. y Scholes, M. (1973). The Pricing of Options and Corporate Liabilities. The Journal of Political Economy, 81(3), 637-654. https://doi.org/10.1086/260062

Blanc-Brude, F., y Hasan, M. (2016). A Structural Model of Credit Risk for Illiquid Debt. The Journal of Fixed Income, 26(1), 6-19. https://doi.org/10.3905/jfi.2016.26.1.006

Blanc-Brude, F., y Hasan, M. (2017). You can work it out! Valuation and recovery of private debt with renegotiable default threshold. The Journal of Fixed Income, 26(4), 113-127. https://doi.org/10.3905/jfi.2017.26.4.113

Blanc-Brude, F., Hasan, M., e Ismail, R (2014). Unlisted Infrastructure Debt Valuation \& Performance Measurement. EDHEC-Risk Institute Publications. Disponible en https://edhec.infrastructure.institute/ wp-content/ uploads/2018/08/24_FINAL_web040914.pdf. Consultado el 1 de febrero de 2019 
Blanc-Brude, F., Hasan, M., y Whittaker, T. (2016). Cash Flow Dynamics of Private Infrastructure Project Debt: Empirical evidence and dynamic modelling. EDHEC-Risk Institute Publications.

Borgonovo, E., y Gatti, S. (2013). Risk analysis with contractual default. Does covenant breach matter? European Journal of Operational Research, 230(2), 431-443. https://doi.org/10.1016/j.ejor.2013.04.047

Cappon, A., Gorenstein, A., Mignot, S., y Manuel, G. (2018). Credit Ratings, Default Probabilities, and Logarithms. The Journal of Structured Finance, 24(1), 39-49. https://doi.org/10.3905/jsf.2018.24.1.039

Cartea, Á. y Figueroa, M. (2005). Pricing in Electricity Markets: A Mean Reverting Jump Diffusion Model with Seasonality. Applied Mathematical Finance, 12(4), 313-335. https://doi.org/10.1080/13504860500117503

Dong, F., Chiara, N., Kokkaew, N., y Xu, A. (2012). Copula-Based Portfolio Credit Risk Assessment in Infrastructure Project Financing. Journal of Private Equity, 15(2), 31-40. https://www.jstor.org/stable/43503709

Freydefont, M. (2001). An Approach to Credit Risk Valuation for Structured and Project Finance Transactions. Journal Of Project Finance, 6(4), 53. https://doi.org/10.3905/ jsf.2001.320236

Gatti, S. (2008). Project finance in theory and practice: designing, structuring, and financing private and public projects. Oxford: Academic Press

Gatti S., Rigamonti A., Saita F. y Senati M. (2007). Measuring Value-at-Risk in project finance transactions. European Financial Management, 13 (1), 135-158. https://doi.org/ 10.1111/j.1468-036X.2006.00288.x

Grimsey, D. y Lewis, M. (2002). Evaluating the risks of public private partnerships for infrastructure projects. International Journal of Project Management, 20(2), 107-118. https://doi.org/10.1016/S0263-7863(00)00040-5

Jobst, A. (2018). Credit Risk Dynamics of Infrastructure Investment: Considerations for Financial Regulators. Washington, D.C: World Bank. http://dx.doi.org/10.2139/ ssrn.3152977

Karminsky, A., y Morgunov, А. (2016). The assessment of the credit risk of investment projects. XVI прельская международная научная конференция по проблемам развития экономики и общества.. Moscú, National Research University Higher School of Economics, 721-731. Disponible en https://publications.hse. ru/en/chapters/180755915. Consultado el 1 de diciembre de 2018.

Kealhofer, S. (1993). Portfolio management of default risk, KMV Corporation

Klompjan, R., y Wouters, M. (2002). Default Risk in Project Finance. The Journal of Structured Finance, 8(3), 10-21. https://doi.org/10.3905/jsf.2002.320283

Kong, D., Tiong, R., Cheah, C., Permana, A., y Ehrlich, M. (2008). Assessment of Credit Risk in Project Finance. Journal of Construction Engineering and Management, 134(11), 876-884. https://doi.org/10.1061/ (ASCE)0733-9364(2008)134:11(876)

McQuown, J. (1993). Market vs. accounting based measures of default risk, KMV Corporation

Merton, R. (1973). Theory of Rational Option Pricing. Bell Journal of Economics and Management Science, 4(1), 141-183. https://doi.org/10.2307/3003143

Merton, R. (1974). On the pricing of corporate debt: The risk structure of interest rates. Journal of Finance, 29, 449-470. https://doi.org/10.1111/j.1540-6261.1974.tb03058.x

Merton, R. (1977). On the pricing of contingent claims and the Modigliani-Miller theorem. Journal of Finance, 29(5), 241-249. https://doi.org/10.1016/0304-405X(77)90020-4

Moody's (2017). Default and Recovery Rates for Project Finance Bank Loans, 1983-2015, Moody's Investor Service: Infrastructure and Project Finance, London, UK

Vasicek, O. (1984). The philosophy of credit valuation: the credit valuation model, KMV Corporation.

Wang, X., Shi, L., Wang, B., y Kan, M. (2019). A method to evaluate credit risk for banks under PPP project finance. Engineering, Construction and Architectural Management. https://doi.org/10.1108/ECAM-06-2018-0247

Yescombe, E. (2002). Principles of Project Finance. San Diego: Academic Press. 